\title{
Design and Fabrication of All Organic Field Effect Transistor
}

\author{
Suprapto* \\ Department of Chemistry, ITS, Surabaya, Indonesia \\ Krishna C. Persaud \\ School of Chemical Eng. and Analytical Science, The University of Manchester, UK
}

\begin{abstract}
All organic field effect transistor consist of poly-3-hexyloxythiophene, undoped poly-3,3'-didecyl-2,2',5',2'terthiophene and polypyrrole has been successfully developed. Poly-3-hexyloxythiophene was applied as gate material. Undoped poly-3,3"-didecyl-2,2',5',2"-terthiophene was used as insulating layer and polypyrrole was applied as source-drain material. The multilayer polymers were deposited onto gold source-drain and gate electrodes by electropolymerization method. The spaces between the gold electrodes were $50 \mu \mathrm{m}$. The transistor shows a current amplification upon increasing gate voltages. Good conductivity stability upon increasing gate voltages was observed. Overall the field effect transistor has properties that similar to inorganic field effect transistor.
\end{abstract}

KEYWORDS: organic field effect transistor, alkylthiophene, alkoxythiophene, electropolymerization

\section{INTRODUCTION}

Poly-3-hexylthiophene has been applied as a gate material in planar field effect transistors and Schottky barrier diode that produce a charge mobility higher than found in the metal oxide transistors [1]. Poly-3-octylthiophene applied as planar field effect transistor show an ideal diode properties in the dark [2]. Quarterthiophene, monohexylquarter-thiophene, dihexyl-quarterthiophene and dihexylsexithiophene applied as gate materials on field effect transistor produce high charge mobility that linear to the long of the alkyl side chains [3]. Poly-3,3"-didodecyl-2,2',5',2"-terthiophene and Poly3,3"-dipentoxy-2,2',5',2"'-ter-thiophene polycrystalline film applied in thin film transistor showlinear response to applied to measure ethanol and 1-hexanol vapors in the 200-2000 ppm ranges [4].

The aims of this experiments are to investigate the characteristics of the organic field effect transistors based on polypyrrole, poly-3-hexyloxythiophene and poly-3,3"didecyl-2,2',5',2'-terthiophene source-drain and gate materials. The insulating layer material used was undoped poly3,3"-didecyl-2,2',5',2"'-terthiophene.

\section{EXPERIMENT}

Field effect transistor configuration used in this experiment is described in figure 1 . The polymer source-drain was set to a high resistance state by dedoping the polymer film. The source-drain semiconductors used were

\footnotetext{
*E-MAIL: suprapto@chem.its.ac.id
}

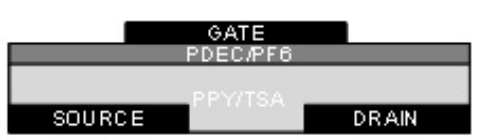

(a)

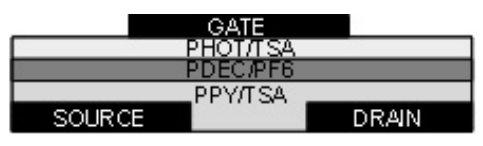

(b)

FIG. 1: FET configurations for PPY/TSA source-drain material and (a) PDEC/PF6 (b) PDEC/PF6-PHOT/TSA gate materials.

polypyrrole and poly-3,3"'-didecyl-2,2',5',2"-terthiophene. The insulating layer used was undoped poly-3,3"-didecyl2,2',5',2"'terthiophene. Gate materials used were polyethylene dioxythiophene (PEDOT), poly-3,3'-didecyl-2,2',5',2"'terthiophene and polypyrrole. The source drain electrode distances were $50 \mu \mathrm{m}$.

The source-drain voltages and source-drain currents were measured using $\mu \mathrm{Lab}$ III EcoChimie Potentiostat with maximum-minimum voltage $\pm 5 \mathrm{~V}$. The gate voltages were generated from DC output of commercial power supply. The source-drain voltage was maintained at $-1 \mathrm{~V}$. The gate voltage applied were $0 \mathrm{~V}, \pm 5 \mathrm{~V}, \pm 10 \mathrm{~V}$ and $\pm 15 \mathrm{~V}$. 


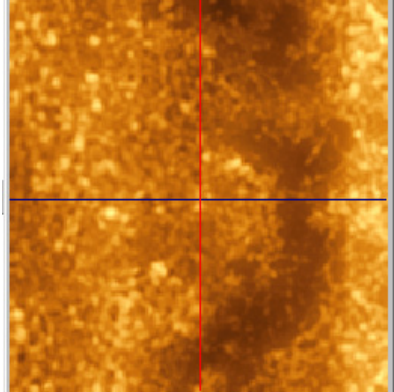

(a)
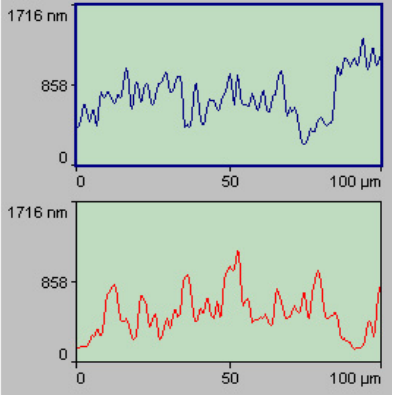

(b)
FIG. 2: Film morphology of PPY/TSA on PDEC/PF6 at $100 \mu^{2}$.

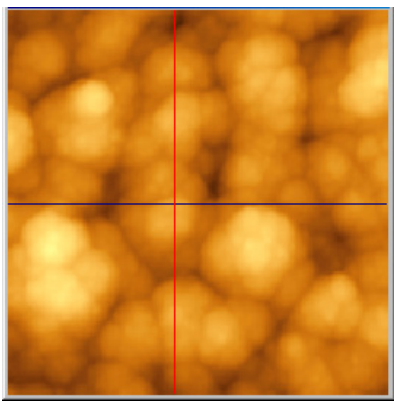

(a)
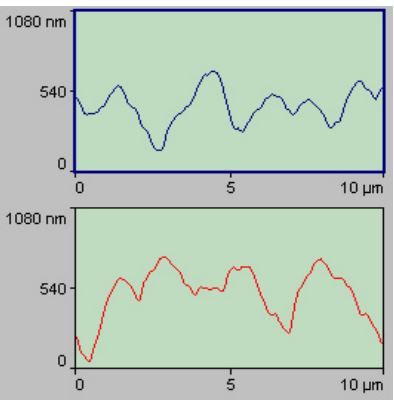

(b)
FIG. 3: Film morphology of PPY/TSA on PDEC/PF6 at $10 \mu^{2}$.

\section{RESULTS AND DISCUSSION}

\section{A. Polypyrrole Gate Material}

Polypyrrole deposited on to poly-3,3"'-didecyl-2,2',5',2"'terthiophene produced a rough and flat film, figure 2 . The gap between source-drain gold electrodes was well covered, a characteristic that was not observed when only polypyrrole was used as base layer. The nodules at $10 \mu \mathrm{m}^{2}$ film were uniform, figure 3.

\section{B. Poly-3-Hexyloxythiophene and}

\section{Poly-3,3"'Didecyl-2,2',5',2'-Terthiophene Gate Materials}

Electrochemical deposition of poly-3-hexyloxythiophene on poly-3,3"-didecyl-2,2',5',2"-terthiophene produces a nodular film, figure 4 . The film morphology was different compared to poly-3-hexyloxythiophene stand-alone film that produce stem like, flat films. Poly-3-hexyloxythiophene on poly-3,3"'-didecyl-2,2',5',2"'-terthiophene at $10 \mathrm{~m} 2$ shows non-uniform nodular film, figure 5.

Noisy source-drain currents were observed when undoped poly-3,3"'-didecyl-2,2',5',2'-terthiophene used as gate material, figure 6. A good sensitivity to ethanol was observed at $-1 \mathrm{~V}$ source-drain voltage. The sensitivity of transistor to pentanol increase as gate voltages increased as shown in figure 7. The source-drain currents-voltage were similar to inorganic

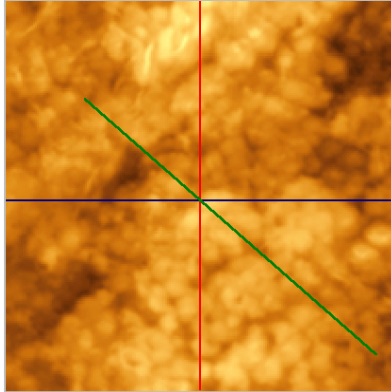

(a)



(b)
FIG. 4: Film morphology of poly-3-hexylthiophene on poly-3,3"didecyl-2,2',5',2"'-terthiophene at $100 \mu^{2}$.

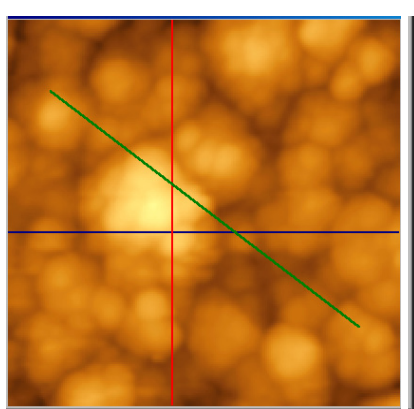

(a)

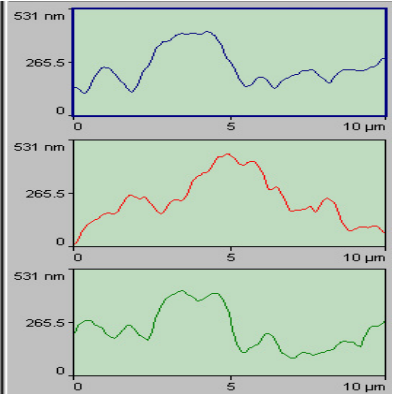

(b)
FIG. 5: Film morphology of poly-3-hexylthiophene on poly-3,3"didecyl-2,2',5',2"'-terthiophene at $10 \mu^{2}$.

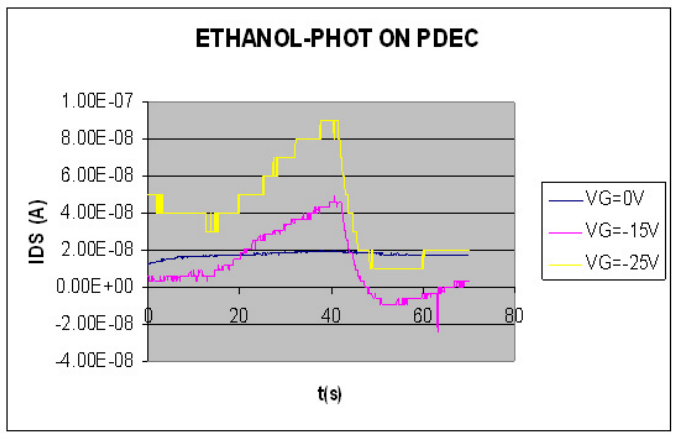

FIG. 6: PHOT/TSA gate material on PPY/TSA source-drain material for dry air and ethanol measurement

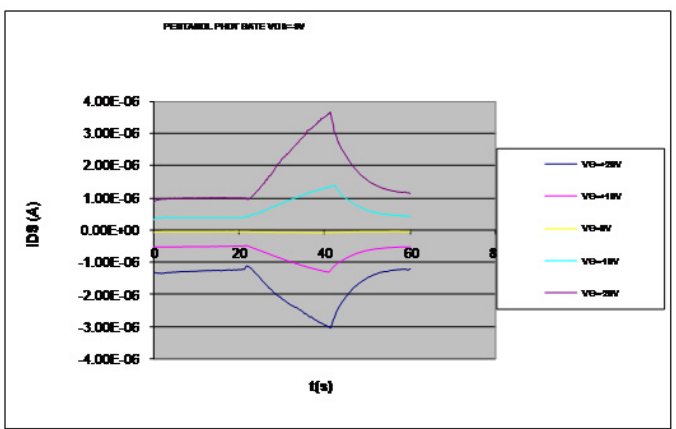

FIG. 7: IDS vs. VDS for PPY/TSA gate on PHOT/TSA source-drain material for pentanol measurement 


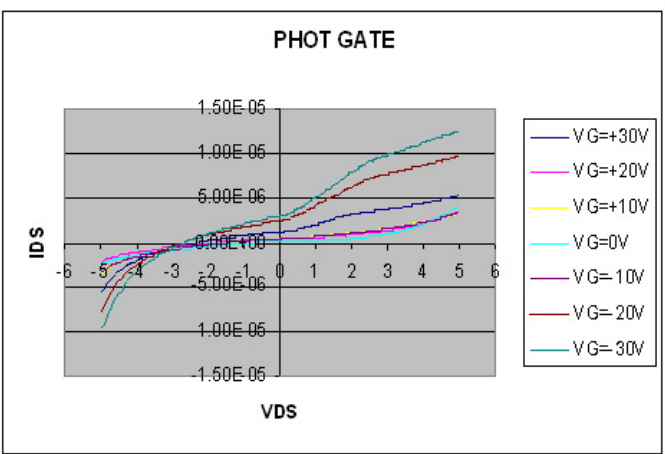

FIG. 8: FET characteristic of PHOT/TSA on PDEC/PF6 field effect transistor characteristics which contain linear and saturated region at various gate voltage applied, as shown in figure 8 .

The on/off voltage and threshold voltage for both transistor configurations were at about $-10 \mathrm{~V}$.

\section{CONCLUSION}

All organic transistors consist of undoped poly-3,3"- didecyl -2,2',5',2"-terthiophene show a current amplification upon increasing gate voltage. Polypyrrole source-drain material shows better stability with increasing gate voltage.
[1] Assadi, A., Et Al., Synthetic Metals, 28(1-2),pp. 863-869 (1989).

[2] Janata, J. and M. Josowicz, Solid State Ionics, 94(1-4), pp.209215 (1997).
[3] Assadi, A., Et Al., Synthetic Metals, 37(1-3), pp.23-130 (1990).

[4] Assadi, A., Et Al., Synthetic Metals, 58(2), pp.187-193 (1993). 